\title{
Bounds on gravitational wave backgrounds from large distance clock comparisons
}

\author{
S. Reynaud* and B. Lamine \\ Laboratoire Kastler Brossel, UPMC, ENS, CNRS, F75252 Paris \\ L. Duchayne and P. Wolf \\ LNE-SYRTE, Observatoire de Paris, CNRS, UPMC, F75014 Paris \\ M.-T. Jaeke \\ Laboratoire de Physique Théorique de l'ENS, CNRS, UPMC, F75231 Paris
}

(Dated: November 10, 2018)

\begin{abstract}
Our spacetime is filled with gravitational wave backgrounds that constitute a fluctuating environment created by astrophysical and cosmological sources. Bounds on these backgrounds are obtained from cosmological and astrophysical data but also by analysis of ranging and Doppler signals from distant spacecraft. We propose here a new way to set bounds on those backgrounds by performing clock comparisons between a ground clock and a remote spacecraft equipped with an ultra-stable clock, rather than only ranging to an onboard transponder. This technique can then be optimized as a function of the signal to be measured and the dominant noise sources, leading to significant improvements on present bounds in a promising frequency range where different theoretical models are competing. We illustrate our approach using the SAGAS project which aims to fly an ultra stable optical clock in the outer solar system.
\end{abstract}

\section{INTRODUCTION}

The basic observables used for synchronizing remote clocks or ranging to distant events are built up on electromagnetic signals exchanged between remote observers and compared to locally available atomic clocks [1]. This statement applies for example to planetary radar ranging [2], lunar laser ranging 3, 4], synchronizing orbiting clocks with Earth-bound standards [5] or tracking and navigating probes in deep space [6].

Electromagnetic links feel the gravitational waves (GW) and this is currently the main route toward GW detection. It follows that GW affect ranging and Doppler tracking observables [7, 8]. This effect has been thoroughly studied in particular with the Pioneer and Cassini probes [9, 10, 11], leading to constraints on the GW noise spectrum in some frequency range [12]. These studies constitute one of the windows on the physics of the stochastic GW backgrounds which permeate our spatiotemporal environment and have an astrophysical or cosmological origin [13, 14, 15, 16, 17]. Their results have to be compared with bounds obtained through different observations [18] (more discussions below).

The aim of the present paper is to show that remote clock synchronization is also affected by GW and might be used to set new bounds. Timing is less sensitive than ranging at distances shorter than the GW wavelength, but this is no longer the case at large distances. Furthermore, the timing procedure can be arranged in order to get rid of uncertainties on the motion of the remote

\footnotetext{
*Electronic address: serge.reynaud@spectro.jussieu.fr

${ }^{\dagger}$ Electronic address: peter.wolf@obspm.fr

‡Electronic address: marc.jaekel@lpt.ens.fr
}

clock, which might greatly improve the bounds on GW at low frequencies. The numbers will be discussed below by taking as an example the SAGAS project which aims at flying ultrastable optical atomic clocks in the outer solar system [19]. These numbers heavily rely on the extremely good accuracy of modern atomic clocks [20, 21, 22].

In the next section, we introduce and compare the basic observables associated with ranging and timing. We then discuss their sensitivity to stochastic GW backgrounds as well as the noise sources involved in their measurement. We finally deduce the constraints on GW backgrounds which could be drawn from comparisons between accurate clocks at large distances from each other in the solar system.

\section{RANGING AND TIMING OBSERVABLES}

We study the comparison between an atomic clock on board a probe and another one colocated with a station on Earth. The clocks are compared using up- as well as down-links. The uplink signal is emitted from ground at positions $\left(t_{1}, \mathbf{x}_{1}\right)$ in time and space and received in space at $\left(t_{2}, \mathbf{x}_{2}\right)$. The downlink signal is emitted from space at $\left(t_{3}, \mathbf{x}_{3}\right)$ and received on ground at $\left(t_{4}, \mathbf{x}_{4}\right)$ (see fig. 1). The up-link is independent from the down-link i.e. $t_{3}-t_{2}$ can be chosen to take any value, positive or negative.

The positions of the emission and reception events are connected by light cones, which may be calculated through a variety of theoretical methods (see for example [23, 24, 25]). These calculations give solutions which depend on the motions of Earth station and space probe, as well as on the gravitational field described by the metric. As the GW are weak modifications of the metric, we treat their effect as a perturbation of the solutions.

These solutions may be written as relations between 


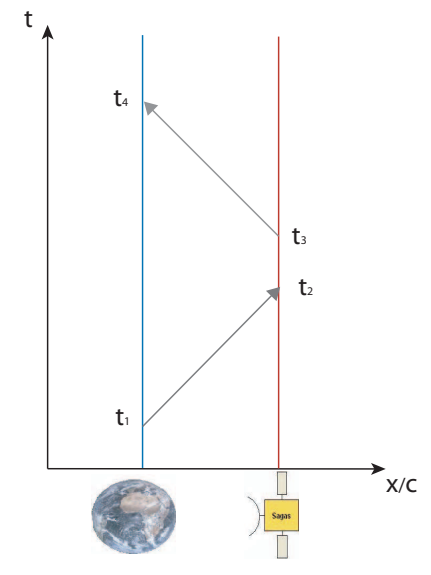

FIG. 1: Principle of a general two-way link with $t_{2} \neq t_{3}$

clock indications corresponding to proper times elapsed on ground or space, say $\tau_{1}^{\mathrm{g}}$ and $\tau_{2}^{\mathrm{s}}$ for the uplink, and $\tau_{3}^{\mathrm{s}}$ and $\tau_{4}^{g}$ for the downlink (see fig. 1). We first define a ranging observable by

$$
\begin{aligned}
\tau_{\mathrm{r}} & \equiv-\frac{\tau_{3}^{\mathrm{s}}-\tau_{2}^{\mathrm{s}}}{2}+\frac{\tau_{4}^{\mathrm{g}}-\tau_{1}^{\mathrm{g}}}{2} \equiv \frac{\tau_{\mathrm{d}}+\tau_{\mathrm{u}}}{2} \\
\tau_{\mathrm{u}} & \equiv \tau_{2}^{\mathrm{s}}-\tau_{1}^{\mathrm{g}} \quad, \quad \tau_{\mathrm{d}} \equiv \tau_{4}^{\mathrm{g}}-\tau_{3}^{\mathrm{s}}
\end{aligned}
$$

In the general case up- and downlinks are defined independently from each other, but a specific configuration of interest is the situation where the links coincide at the space endpoint $\left(\tau_{2}^{\mathrm{s}}=\tau_{3}^{\mathrm{s}} \Leftrightarrow t_{2}=t_{3}\right)$. Then $\tau_{\mathrm{r}}$ represents a spatial distance corresponding to half the proper time elapsed on ground during the roundtrip of the signal to and from the probe. This special case is the only one realized in deep space probes so far, which were only equipped with a transponder that essentially only reflects the incoming signal. The range observable (1) is unaffected at lowest order by clock uncertainties in space (when using $t_{2}=t_{3}$ ) and can even be measured without a good clock on board.

Another observable of great interest is the combination of $\tau_{\mathrm{u}}$ and $\tau_{\mathrm{d}}$ defined with the opposite sign

$$
\tau_{\mathrm{t}} \equiv \frac{\tau_{3}^{\mathrm{s}}+\tau_{2}^{\mathrm{s}}}{2}-\frac{\tau_{4}^{\mathrm{g}}+\tau_{1}^{\mathrm{g}}}{2} \equiv \frac{-\tau_{\mathrm{d}}+\tau_{\mathrm{u}}}{2}
$$

This timing observable (2) is unaffected at lowest order by uncertainties in the motion of the probe (when $t_{2}=$ $t_{3}$ ). For a probe equipped with a clock and a two-way system, one can choose to use either (or both) of the observables (11) and (2) with a free choice of the value of $t_{3}-t_{2}$ in order to optimize the measurement depending on the signal to be measured and the noise affecting the measurements. This is not the case for probes equipped only with a transponder, which are limited to the special case of (1) with $t_{2}=t_{3}$.

We also introduce the time derivatives of (1) and (2)

$$
y_{\mathrm{r}} \equiv \dot{\tau}_{\mathrm{r}}=\frac{y_{\mathrm{d}}+y_{\mathrm{u}}}{2} \quad, \quad y_{\mathrm{t}} \equiv \dot{\tau}_{\mathrm{t}}=\frac{-y_{\mathrm{d}}+y_{\mathrm{u}}}{2}
$$

The dot symbol represents here a derivation with respect to a commonly defined time $t$, chosen for any convenient argument. Note that $y_{\mathrm{r}}$ is directly related to the Doppler tracking observable, which has been over the years the main source of information on the navigation of remote probes [26]. Meanwhile $y_{\mathrm{t}}$ is directly related to the frequency comparison of distant clocks, the so-called syntonization observable. The variations of these quantities can be evaluated in the framework of a linearized approximation with a reasonably good approximation in the solar system. A more precise evaluation would be easy by using available methods [23] and it would not change the qualitative discussions presented below.

The variations in (31) are due to the effect of motion of the probe and ground station and perturbing effects like atmospheric delays, clock noise, etc... (see sect. 4) on one hand, and to the integrated effect of gravity along the propagation of the electromagnetic link on the other hand. The latter is given by an integral along the up- or downlink paths $[\mathrm{u}]$ and $[\mathrm{d}]$

$$
\delta \tau_{\mathrm{u}, \mathrm{d}}=-\frac{1}{2 c} \int_{[\mathrm{u}, \mathrm{d}]} h_{i j}^{\mathrm{TT}} \frac{\mathrm{d} x_{\mathrm{u}, \mathrm{d}}^{i}}{\mathrm{~d} \sigma} \frac{\mathrm{d} x_{\mathrm{u}, \mathrm{d}}^{j}}{\mathrm{~d} \sigma} \mathrm{d} \sigma
$$

$h_{i j}^{\mathrm{TT}}$ is the metric perturbation $\left(h_{\mu \nu} \equiv g_{\mu \nu}-\eta_{\mu \nu}\right.$ with $g_{\mu \nu}$ and $\eta_{\mu \nu}$ the metric and Minkowski tensors) in the transverse traceless (TT) gauge; $\sigma$ is the affine parameter along the path measured as a length, and $\frac{\mathrm{d} x}{\mathrm{~d} \sigma}$ the electromagnetic wavevector reduced so that its time component is unity.

\section{SENSITIVITY TO STOCHASTIC GW BACKGROUNDS}

We now evaluate the effect of stochastic GW backgrounds as sources of noise on the electromagnetic links. For simplicity, we consider the background to be isotropic and unpolarized. We first recover well known results for the ranging case with $t_{2}=t_{3}$ and then discuss the timing observable and the general case $t_{2} \neq t_{3}$.

To this aim, we introduce a plane wave decomposition of the GW background with wavevector $\mathbf{k}=k \mathbf{n}(k=\omega / c$ the modulus of $\mathbf{k}$ and $\mathbf{n}$ its direction)

$$
h_{i j}^{\mathrm{TT}}(t, \mathbf{x})=\int \frac{\mathrm{d}^{3} \mathbf{k}}{(2 \pi)^{3}} h_{i j}^{\mathrm{TT}}[k \mathbf{n}] e^{-i \omega t+i \mathbf{k} \cdot \mathbf{x}}+c . c .
$$

We then write the Fourier component $\delta \tau[\omega]$ of the phase shifts appearing in eq.(4). At each frequency $\omega$, this component is an integral over the direction $\mathbf{n}$ of GW plane waves with wavevectors $\mathbf{k}=(\omega / c) \mathbf{n}$ weighted by sensitivity amplitudes. Considering as an example propagation along axis 1, we get

$$
\delta \tau[\omega]=-\frac{\omega}{2 \pi c^{3}} \int \frac{\mathrm{d}^{2} \mathbf{n}}{4 \pi} \frac{h_{11}^{\mathrm{TT}}[k \mathbf{n}]}{1-\mu^{2}} \beta[k \mathbf{n}]
$$


The sensitivity amplitudes $\beta[k \mathbf{n}]$ depend on the frequency $\omega$ and the parameter $\mu \equiv \mathbf{n}_{1}$, which is the component of $\mathbf{n}$ along the direction of propagation of the electromagnetic signal (here the axis 1 ). For a signal emitted at $\left(t_{1}, \mathbf{x}_{1}\right)$ and received at $\left(t_{2}, \mathbf{x}_{2}\right)$ (uplink on figure 1 ) the sensitivity amplitude is given by [27]

$$
\beta[k \mathbf{n}]=\frac{1+\mu}{-i}\left(e^{-i \omega t_{2}} e^{i \mathbf{k} \cdot \mathbf{x}_{2}}-e^{-i \omega t_{1}} e^{i \mathbf{k} \cdot \mathbf{x}_{1}}\right)
$$

We will consider for simplicity the case of a stationary, unpolarized and isotropic background. The background may thus be characterized by a spectral density $S_{\mathrm{GW}}[\omega]$ giving the strain noise at a space point $\mathbf{x}$ [28]

$$
\left\langle h_{11}^{\mathrm{TT}}(t, \mathbf{x}) h_{11}^{\mathrm{TT}}(0, \mathbf{x})\right\rangle=\frac{4}{3} \int_{0}^{\infty} \frac{\mathrm{d} \omega}{2 \pi} S_{\mathrm{GW}}[\omega] \cos (\omega t)
$$

The fluctuations of $\tau$ are finally characterized by a noise spectrum $S_{\tau}[\omega]$ such that 28$]$

$$
\begin{aligned}
& \langle\delta \tau(t) \delta \tau(0)\rangle=\int_{0}^{\infty} \frac{\mathrm{d} \omega}{2 \pi} S_{\tau}[\omega] \cos (\omega t) \\
& S_{\tau}[\omega]=\frac{5}{8 \omega^{2}} b[\omega] S_{\mathrm{GW}}[\omega]
\end{aligned}
$$

The dimensionless function $b$ is obtained by averaging $|\beta[k \mathbf{n}]|^{2}$ over the direction $\mathbf{n}$ of the $\mathrm{GW}$ wavevector

$$
b[\omega] \equiv\left\langle|\beta[k \mathbf{n}]|^{2}\right\rangle_{\mathbf{n}}=\int_{-1}^{+1} \frac{\mathrm{d} \mu}{2}|\beta[k \mathbf{n}]|^{2}
$$

\section{A. Up- and downlinks with $t_{2}=t_{3}$}

We first discuss the special case $t_{2}=t_{3}$. The sensitivity amplitudes for the up- and down- links are obtained directly from (7) by fixing the origin of coordinates $t_{2}=t_{3}=\mathbf{x}_{2}=\mathbf{x}_{3}=0$ yielding

$$
\begin{aligned}
& \beta_{\mathrm{u}}=\frac{1+\mu}{-i}\left(1-e^{i(1-\mu) \omega T}\right) \\
& \beta_{\mathrm{d}}=\frac{1-\mu}{i}\left(1-e^{-i(1+\mu) \omega T}\right)
\end{aligned}
$$

We have introduced shorthand notations for the propagation time $T$ and the cosine $\mu$ (defined for the uplink)

$$
T \equiv t_{2}-t_{1}=t_{4}-t_{3}
$$

Throughout the paper we assume that the relative motion of the space probe and the Earth during signal propagation is negligible (so that $t_{2}-t_{1}=t_{4}-t_{3}$ ). More general expressions for the situation where this is not the case can also be obtained, but are beyond the scope of this work. The up- and downlink expressions are exchanged by taking opposite signs for the cosine $\mu$, the propagation time $T$ and the global expression.

The resulting $b$-function is already known 27]

$$
b \equiv b_{\mathrm{u}}=b_{\mathrm{d}}=2\left(\frac{4}{3}+\frac{\sin (2 \omega T)-2 \omega T}{(\omega T)^{3}}\right)
$$

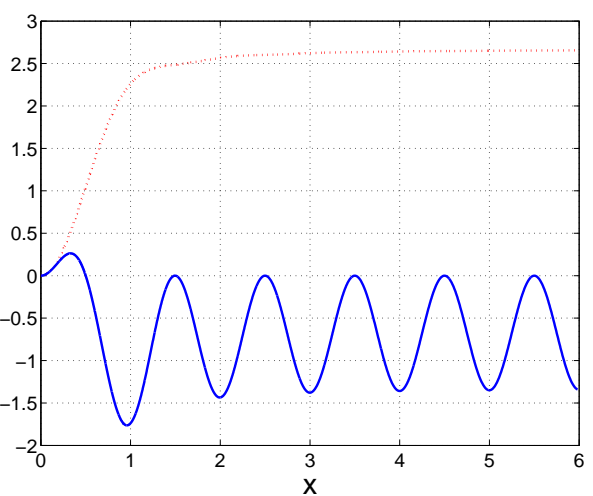

FIG. 2: $b$ (red-dotted) and $c$ (blue-solid) as functions of $x \equiv$ $\omega T / \pi$

For obvious symmetry reasons, it has the same form for up- and downlinks.

The variation of $b$ versus the dimensionless parameter $x \equiv \omega T / \pi$ is shown as the red-dotted curve on Figure 2 . The blue-solid curve on Figure 2 represents the covariance function $c$ which describes the correlation of the upand downlinks (* denotes complex conjugation)

$$
c[\omega] \equiv \frac{1}{2}\left\langle\beta_{\mathrm{u}}[k \mathbf{n}] \beta_{\mathrm{d}}^{*}[k \mathbf{n}]+\beta_{\mathrm{d}}[k \mathbf{n}] \beta_{\mathrm{u}}^{*}[k \mathbf{n}]\right\rangle_{\mathbf{n}}
$$

The average over $\mathbf{n}$ can be evaluated as

$$
\begin{aligned}
& c[\omega]=-4 \gamma[\omega] \cos (\omega T) \\
& \gamma[\omega] \equiv \frac{\cos (\omega T)}{3}+\frac{\cos (\omega T)}{(\omega T)^{2}}-\frac{\sin (\omega T)}{(\omega T)^{3}}
\end{aligned}
$$

We conclude this section by discussing qualitatively the shapes of the two curves $b$ and $c$. We first notice that $\beta_{\mathrm{u}}$ and $\beta_{\mathrm{d}}$ tend to become identical at the limit of short distances or low frequencies $\left(\beta_{\mathrm{u}} \simeq \beta_{\mathrm{d}} \simeq\left(1-\mu^{2}\right) \omega T\right.$ for $\omega T \ll 1)$, so that $b$ and $c$ show the same behaviour $\simeq \frac{8}{15} \omega^{2} T^{2}$. The noise spectrum (9) is thus reduced to the simple form (corresponding to $\delta \tau_{\mathrm{u}} \simeq \delta \tau_{\mathrm{d}} \simeq-\frac{T}{2} h_{11}$ )

$$
S_{\tau}[\omega]=\frac{T^{2}}{3} S_{\mathrm{GW}}[\omega] \quad, \quad \omega T \ll 1
$$

At the high-frequency or large distance limit $\omega T \gg 1$ in contrast, $b$ goes to a constant so that

$$
S_{\tau}[\omega]=\frac{5}{3 \omega^{2}} S_{\mathrm{GW}}[\omega] \quad, \quad \omega T \gg 1
$$

Meanwhile, the correlation $c$ between up- and downlinks remains sensitive to the distance even at large distances. This is simply due to the fact that $\left(\beta_{\mathrm{u}} \beta_{\mathrm{d}}^{*}+\beta_{\mathrm{d}} \beta_{\mathrm{u}}^{*}\right) / 2$ contains a part $\left(\mu^{2}-1\right)(1+\cos (2 \omega T))$ which is not blurred by the integration over $\mu$. We also note that $c$, which is positive at low frequencies $(\omega T \ll 1)$, is negative at high frequencies $\left(\omega T>\frac{\pi}{2}\right)$. 


\section{B. Ranging and timing with $t_{2}=t_{3}$}

We repeat now the same discussion in terms of the ranging and timing observables. It is clear from (11) and (2) that one can write expressions similar to (6) for $\delta \tau_{\mathrm{r}}$ and $\delta \tau_{\mathrm{t}}$ with the following sensitivity amplitudes

$$
\beta_{\mathrm{r}}=\frac{\beta_{\mathrm{u}}+\beta_{\mathrm{d}}}{2} \quad, \quad \beta_{\mathrm{t}}=\frac{-\beta_{\mathrm{u}}+\beta_{\mathrm{d}}}{2}
$$

The noise spectra have the same form as (9) with the sensitivity functions

$$
b_{\mathrm{r}}[\omega]=\left\langle\left|\beta_{\mathrm{r}}[k \mathbf{n}]\right|^{2}\right\rangle_{\mathbf{n}} \quad, \quad b_{\mathrm{t}}[\omega]=\left\langle\left|\beta_{\mathrm{t}}[k \mathbf{n}]\right|^{2}\right\rangle_{\mathbf{n}}
$$

which can be written in terms of the already discussed functions $b$ and $c$

$$
b_{\mathrm{r}}[\omega]=\frac{b[\omega]+c[\omega]}{2} \quad, \quad b_{\mathrm{t}}[\omega]=\frac{b[\omega]-c[\omega]}{2}
$$

It has also to be stressed that the correlation between the ranging and timing variables vanishes, as can be shown through an explicit calculation. As a matter of fact, the sensitivity amplitudes can be written as

$$
\begin{aligned}
\beta_{\mathrm{r}}= & (\sin \omega T-\mu \sin \mu \omega T \\
& -i \mu(\cos \omega T-\cos \mu \omega T)) \exp ^{-i \mu \omega T} \\
\beta_{\mathrm{t}}= & (\sin \mu \omega T-\mu \sin \omega T \\
& +i(\cos \omega T-\cos \mu \omega T)) \exp ^{-i \mu \omega T}
\end{aligned}
$$

and it turns out that $\left(\beta_{\mathrm{r}} \beta_{\mathrm{t}}^{*}+\beta_{\mathrm{t}} \beta_{\mathrm{r}}^{*}\right) / 2$ is odd in $\mu$ and vanishes after the angular integration. Alternatively the fact that the correlation between $\delta \tau_{\mathrm{r}}$ and $\delta \tau_{\mathrm{t}}$ vanishes can be directly inferred from the already discussed property $b_{\mathrm{u}}=b_{\mathrm{d}}$, which was attributed to a symmetry between up- and downlinks. It means that $\delta \tau_{\mathrm{r}}$ and $\delta \tau_{\mathrm{t}}$ appear as intrinsic and independent stochastic fluctuations of the positions in space-time of the end-points.

The explicit expressions of the functions $b_{\mathrm{r}}$ and $b_{\mathrm{t}}$ can be obtained through an explicit calculation or alternatively deduced from (13) and (15)

$$
\begin{aligned}
& b_{\mathrm{r}}[\omega]=\frac{3-\cos (2 \omega T)}{3}-\frac{3+\cos (2 \omega T)}{(\omega T)^{2}}+\frac{2 \sin (2 \omega T)}{(\omega T)^{3}} \\
& b_{\mathrm{t}}[\omega]=\frac{5+\cos (2 \omega T)}{3}-\frac{1-\cos (2 \omega T)}{(\omega T)^{2}}
\end{aligned}
$$

The associated plots are shown for $b_{\mathrm{r}}$ (blue-solid) and $b_{\mathrm{t}}$ (green-dashed) on Figure 3 .

Since $\beta_{\mathrm{u}}$ and $\beta_{\mathrm{d}}$ tend to become identical at the limit of short distances or low frequencies, it follows that the GW affect essentially the ranging observable. We effectively obtain in the limit $\omega T \ll 1$ a much larger value for $b_{\mathrm{r}} \simeq 8(\omega T)^{2} / 15$ than for $b_{\mathrm{t}} \simeq 2(\omega T)^{4} / 15$. This is obviously the reason why this case has been much more studied than the timing case. But is also clear that this is no longer the case for arbitrary frequencies. In particular we know that $c$ is negative at frequencies $\omega T>\frac{\pi}{2}$ so

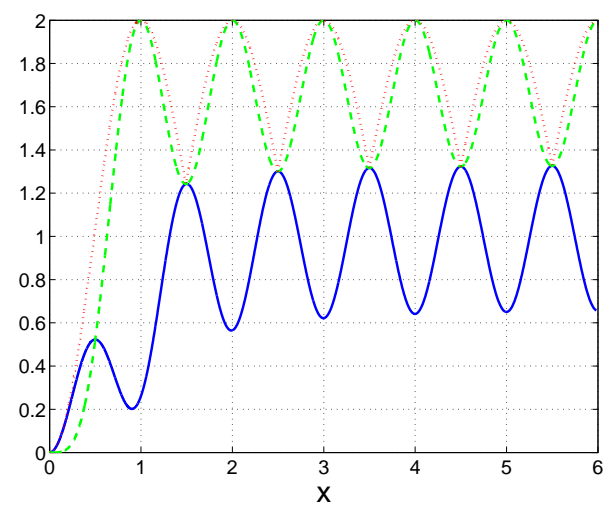

FIG. 3: $b_{\mathrm{r}}$ (blue-solid), $b_{\mathrm{t}}$ (green-dashed) and $b_{\text {opt }}$ (red-dotted, see section 3.3) as functions of $x \equiv \omega T / \pi$

that $b_{\mathrm{t}}$ is larger than $b_{\mathrm{r}}$ in this frequency range. We also notice that the oscillations in $b_{\mathrm{r}}$ and $b_{\mathrm{t}}$ persist at large frequencies or large distances with simple behaviours $b_{\mathrm{r}} \simeq 1-\cos (2 \omega T) / 3$ and $b_{\mathrm{t}} \simeq 5 / 3+\cos (2 \omega T) / 3$ at $\omega T \gg 1$. While this merely reflects the discussion already devoted to $c$ it is worth noting that the oscillations tend to disappear in the sum of $b_{\mathrm{r}}$ and $b_{\mathrm{t}}$ that is also the single-link expression $b$.

\section{General case $t_{2} \neq t_{3}$}

For a spacecraft equipped only with a transponder the only observable that can be obtained is the ranging $\tau_{\mathrm{r}}$ (or its derivative $y_{\mathrm{r}}$ ) for the special case $t_{2}=t_{3}$ (cf Fig. 1). An onboard clock enables one to use also the timing observable $\tau_{\mathrm{t}}$, and additionally provides the possibility to freely choose the ground and onboard measurements, which are combined to form $\tau_{\mathrm{r}}$ or $\tau_{\mathrm{t}}$, ie. to freely choose the value of $t_{3}-t_{2}$. We will show below that this choice can be used to optimize the sensitivity of the observables to a particular signal (in the present case the GW background) for a given measurement noise spectrum.

The general expression (7) for the one-way sensitivity amplitude can be used directly in (18) and (19), to obtain the sensitivity functions $b_{\mathrm{r}, \mathrm{t}}[\omega]$ for an up- and downlink separated by $T_{23} \equiv t_{3}-t_{2}$

$$
\begin{aligned}
& b_{\mathrm{r}}[\omega]=\frac{b}{2}-2 \gamma \cos \left(\omega T_{23}+\omega T\right) \\
& b_{\mathrm{t}}[\omega]=\frac{b}{2}+2 \gamma \cos \left(\omega T_{23}+\omega T\right)
\end{aligned}
$$

with $b$ given in (13) and $\gamma$ in (15). The special case $t_{2}=t_{3}$ (equations (22) ) is recovered when setting $T_{23}=0$ in eq.(23). These general sensitivity functions can now be used in the data analysis to choose the optimum value of $T_{23}$ for each frequency $\omega$ as a function of the link noise and signal travel time $T$. Figure 3 shows the optimized sensitivity function $b_{\text {opt }}$, calculated by maximising either of the equations (23). One clearly obtains $b_{\mathrm{opt}} \geq b_{\mathrm{r}}, b_{\mathrm{t}}$. 


\section{MEASUREMENT NOISE}

In the following, we take the SAGAS (Search for Anomalous Gravity using Atomic Sensors) project [19] as an example to illustrate the advantages and versatility provided by missions with an onboard clock and independent up- and downlinks. The SAGAS project proposes to fly a highly stable and accurate optical atomic clock and atomic accelerometer on an escape orbit in the solar system, up to a distance of $50 \mathrm{AU}$ and beyond. It will use a continuous optical link for clock comparison, navigation, and data transfer, together with an X-band radio link as a backup. Science objectives are centered on tests of fundamental physics, in particular gravity on solar system scales and the exploration of the outer solar system, in particular the Kuiper belt.

The optical link uses continuous transmission of a laser at $f_{0} \approx 444 \mathrm{THz}$ in both directions (up and down) with $1 \mathrm{~W}$ at emission, a $40 \mathrm{~cm}$ telescope on board the satellite and $1.5 \mathrm{~m}$ telescopes on the ground. Numerous perturbations on the link (atmospheric and instrumental losses, received photon flux and shot noise, stray light, etc...) are discussed in [19], section 3.3.4. The fundamental science measurements of SAGAS are the frequency difference between a local laser (optical clock) and an incoming laser beam at the same nominal frequency $f_{0}$, both on board (up-link) and on the ground (down-link), sampled at 0.01 $\mathrm{Hz}$ (see fig. 1). The measurements thus correspond to the observables $y_{\mathrm{u}}$ and $y_{\mathrm{d}}$ defined in (3). Including only terms whose noise contribution plays a significant role they can be expressed as

$$
\begin{aligned}
y_{\mathrm{u}}= & \frac{f_{\mathrm{s}}\left(t_{2}\right)-f_{\mathrm{g}}\left(t_{1}\right)}{f_{0}}+\mathbf{N}_{\mathrm{u}} \cdot \frac{\mathbf{v}_{\mathrm{s}}\left(t_{2}\right)-\mathbf{v}_{\mathrm{g}}\left(t_{1}\right)}{c} \\
& +\Delta y_{\text {tropo }}\left(t_{1}\right) \\
y_{\mathrm{d}}= & \frac{f_{\mathrm{g}}\left(t_{4}\right)-f_{\mathrm{s}}\left(t_{3}\right)}{f_{0}}+\mathbf{N}_{\mathrm{d}} \cdot \frac{\mathbf{v}_{\mathrm{s}}\left(t_{3}\right)-\mathbf{v}_{\mathrm{g}}\left(t_{4}\right)}{c} \\
& +\Delta y_{\text {tropo }}\left(t_{4}\right)
\end{aligned}
$$

where $f_{\mathrm{s}, \mathrm{g}}$ are the frequencies of the space/ground laser (optical clock), $\mathbf{v}_{\mathrm{s}, \mathrm{g}}$ the associated velocity vectors, $\mathbf{N}_{\mathrm{u}, \mathrm{d}}$ the direction vectors of up- and down$\operatorname{links}\left(\mathbf{N}_{\mathrm{u}} \equiv \frac{\mathbf{x}_{\mathrm{s}}\left(t_{2}\right)-\mathbf{x}_{\mathrm{g}}\left(t_{1}\right)}{\left\|\mathbf{x}_{\mathrm{s}}\left(t_{2}\right)-\mathbf{x}_{\mathrm{g}}\left(t_{1}\right)\right\|}, \mathbf{N}_{\mathrm{d}} \equiv \frac{\mathbf{x}_{\mathrm{s}}\left(t_{3}\right)-\mathbf{x}_{\mathrm{g}}\left(t_{4}\right)}{\left\|\mathbf{x}_{\mathrm{s}}\left(t_{3}\right)-\mathbf{x}_{\mathrm{g}}\left(t_{4}\right)\right\|}\right)$, and $\Delta y_{\text {tropo }}$ the frequency change of the signal due to it crossing the Earth's troposphere.

The noise coming from the different terms in (24) can be described equivalently by a power spectral density (PSD) $S_{y}(f)$ or an Allan variance $\sigma_{y}(\tau)$. In the following we consider the simple cases of a white frequency noise (terms proportional to $h_{0}$ ) and of a flicker frequency noise (terms proportional to $h_{-1}$ ) with the translation rule [29]

$$
\begin{aligned}
S_{y}(f) & =\left(h_{0} f^{0}+h_{-1} f^{-1}\right) / \mathrm{Hz} \\
\sigma_{y}^{2}(\tau) & =\frac{h_{0}}{2 \tau}+2 \ln 2 h_{-1}
\end{aligned}
$$

Here the frequency $f$ is in $\mathrm{Hz}$ and the integration time $\tau$ in s (we have kept the notations $h_{0}$ and $h_{-1}$ used in time and frequency metrology and which should not be confused with the metric perturbations $h_{i j}^{\mathrm{TT}}$ ). We first discuss the different noise contributions on a single link and then evaluate the noise on the combined observables $y_{\mathrm{r}}$ and $y_{\mathrm{t}}$ defined in (3) for the general case $t_{2} \neq t_{3}$.

\section{A. One-way link measurement noise}

The space clock fractional frequency stability, as specified in [19], corresponds to a white frequency noise $\sigma_{y}(\tau)=1 \times 10^{-14} \tau^{-1 / 2}$ and an accuracy of $\sigma_{y}(\tau)=$ $1 \times 10^{-17}$, ie. a flicker frequency noise at or below that level. The fractional frequency power spectral density (PSD) is then

$$
S_{y_{s}}(f)=\left(2 \times 10^{-28}+7.2 \times 10^{-35} f^{-1}\right) / \mathrm{Hz}
$$

Though the flicker noise is likely to be lower than the projected accuracy, we use this conservative estimate.

The ground clock stability is likely to be significantly better than the space clock stability by the time the mission is launched. Best present stabilities of optical frequency standards are already below $\sigma_{y}(\tau)=3 \times$ $10^{-15} \tau^{-1 / 2}$ [22], with accuracies at $3 \times 10^{-17}$ [21]. Further rapid improvement of these numbers is expected. We therefore estimate the ground clock noise by the time of mission operation at $\sigma_{y}(\tau)=5 \times 10^{-16} \tau^{-1 / 2}$ with a flicker component at $3 \times 10^{-18}$, so that the PSD is read

$$
S_{y_{g}}(f)=\left(5 \times 10^{-31}+6.5 \times 10^{-36} f^{-1}\right) / \mathrm{Hz}
$$

The noise on the spacecraft velocity of SAGAS is determined by the integrated noise of the on-board accelerometer. Although orbit modeling is likely to improve on the raw accelerometer noise at low frequency, we use that as our conservative estimate for the purpose of this work. The accelerometer noise specified in [19] is $\sigma_{a}(\tau)=9 \times 10^{-10} \tau^{-1 / 2} \mathrm{~m} / \mathrm{s}^{2}$ per axis for $3 \mathrm{D}$ measurements and $\sqrt{3}$ less when measuring only along the direction of signal transmission, of interest here. An absolute accelerometer based on cold atom technology is used to avoid long term drifts and biases (see [19], section 3.1 for details). The expected absolute accuracy is $5 \times 10^{-12} \mathrm{~m} / \mathrm{s}^{2}$ taken again as the upper limit of the flicker acceleration noise. This translates into a velocity PSD of

$$
S_{v_{\mathrm{s}} / c}=\left(1.5 \times 10^{-37} f^{-2}+5.1 \times 10^{-42} f^{-3}\right) / \mathrm{Hz}
$$

For radio-frequency Doppler ranging, one of the dominant noise sources at low frequency is the uncorrected motion of the $34 \mathrm{~m}$ DSN antenna and of the station location itself [30]. For the optical link ground telescopes the motion of the mirror is likely to cause less of a problem, however the site movement plays a similar role as in the radio-frequency case. At high frequencies the motion of the ground station can be corrected using gravity measurements, with best presently achieved 
measurement noise levels of about $4 \times 10^{-18} \mathrm{~m}^{2} / \mathrm{s}^{4} / \mathrm{Hz}$ when using superconducting gravimeters 31. Alternatively, positioning using global navigation systems (GNSS) and/or Satellite Laser Ranging (SLR) and/or Very Long Baseline Interferometry (VLBI) achieve sub$\mathrm{cm}$ uncertainties [32]. Typically, GNSS positioning shows flicker noise in position over a wide range of frequencies [33, 34, 35], with best results at present at about $S_{x}(f)=1 \times 10^{-6} f^{-1} \mathrm{~m}^{2} / \mathrm{Hz}$. The noise level shows some dependence on the number of visible satellites, and therefore further improvement is expected with upcoming additional GNSS systems [36]. SLR and VLBI show white positioning noise [34], but at higher levels than GNSS at the frequencies of interest here $\left(10^{-6}-10^{-5} \mathrm{~Hz}\right)$. Combining local gravity measurements with GNSS positioning we obtain as the minimum noise on ground velocity

$$
\begin{aligned}
& S_{v_{\mathrm{g}} / c}=1.1 \times 10^{-36} \frac{f^{-2}}{\mathrm{~Hz}}, \quad f>1.4 \times 10^{-5} \mathrm{~Hz} \\
& S_{v_{\mathrm{g}} / c}=4.4 \times 10^{-22} \frac{f}{\mathrm{~Hz}}, \quad f \leq 1.4 \times 10^{-5} \mathrm{~Hz}
\end{aligned}
$$

In (29) we have assumed that superconducting gravimeters display white noise down to $10^{-5} \mathrm{~Hz}$, whereas the spectra shown in 31] only show white noise down to $10^{-3} \mathrm{~Hz}$, with the measurements being dominated by natural gravity fluctuations (the signal to be measured) at lower frequencies. This has to be considered as a preliminary estimation of low frequency noise unknown at present.

Typically tropospheric delay models at optical frequencies have millimetric accuracy. Furthermore mapping functions that determine the variation of the delay with elevation (of interest here, the constant part of the delay playing no role on the frequency measurement) have been developed to sub-millimeter accuracy and successfully tested on SLR data [37]. Assuming that the residuals from such models show white phase noise at the $1 \mathrm{~mm}$ level at $10 \mathrm{~Hz}$ sampling (typical pulse rate of SLR and LLR stations) we obtain a frequency PSD of

$$
S_{y_{\text {tropo }}}(f)=8.7 \times 10^{-23} f^{2} / \mathrm{Hz}
$$

Note, that we pessimistically ignore correlations at high frequencies, which, given the slow motion of the satellite in the sky, should lead to decreased high frequency noise. Figure 4 summarizes the noise sources on a one way link discussed above.

\section{B. Measurement noise on ranging and timing}

The noise on the ranging and timing observables defined in (3) is a combination of the noise affecting the individual links, which are clearly correlated when forming the ranging or timing observables. Taking into account

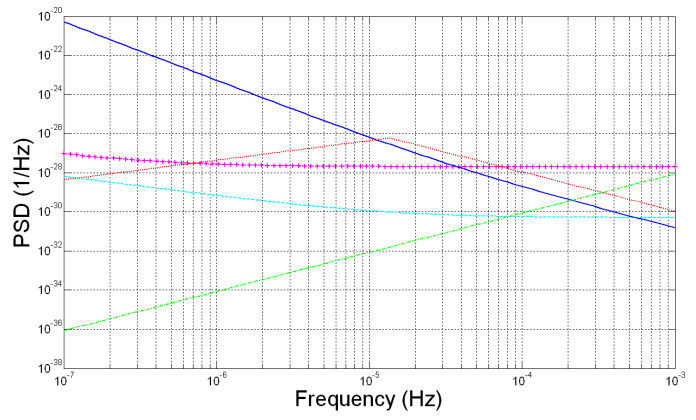

FIG. 4: Power spectral densities of the dominant noise sources on a one-way link: $S_{y_{s}}$ (magenta-crosses), $S_{y_{g}}$ (light bluedashed), $S_{v_{\mathrm{s}} / c}$ (dark blue-solid), $S_{v_{\mathrm{g}} / c}$ (red-dotted), $S_{y_{\text {tropo }}}$ (green-dash-dotted).

those correlations for the general case $t_{2} \neq t_{3}$ we obtain

$$
\begin{aligned}
S_{y_{\mathrm{r}}}(f)=\frac{1}{2} \quad & \left\{\left(1-\cos \left(2 \pi f T_{14}\right)\right) S_{y_{\mathrm{g}}}(f)\right. \\
& +\left(1-\cos \left(2 \pi f T_{23}\right)\right) S_{y_{\mathrm{s}}}(f) \\
& +\left(1+\cos \left(2 \pi f T_{23}\right)\right) S_{v_{\mathrm{s}} / c}(f) \\
& +\left(1+\cos \left(2 \pi f T_{14}\right)\right) S_{v_{\mathrm{g}} / c}(f) \\
& \left.+\left(1+\cos \left(2 \pi f T_{14}\right)\right) S_{y_{\text {tropo }}}(f)\right\} \\
S_{y_{\mathrm{t}}}(f)=\frac{1}{2} \quad & \left\{\left(1+\cos \left(2 \pi f T_{14}\right)\right) S_{y_{\mathrm{g}}}(f)\right. \\
& +\left(1+\cos \left(2 \pi f T_{23}\right)\right) S_{y_{\mathrm{s}}}(f) \\
& +\left(1-\cos \left(2 \pi f T_{23}\right)\right) S_{v_{\mathrm{s}} / c}(f) \\
& +\left(1-\cos \left(2 \pi f T_{14}\right)\right) S_{v_{\mathrm{g}} / c}(f) \\
& \left.+\left(1-\cos \left(2 \pi f T_{14}\right)\right) S_{y_{\text {tropo }}}(f)\right\}
\end{aligned}
$$

where we have defined $T_{i j} \equiv t_{j}-t_{i}$ (cf. fig. 11).

Figures 5 show the resulting noise PSD of the timing and ranging observables for the special cases $T_{23}=0$ (coincidence of up and down signals at the satellite) and $T_{14}=0$ (coincidence of up and down signals on the ground). More generally, (31) and (32) can be used to obtain the noise spectra of $y_{\mathrm{r}}$ and $y_{\mathrm{t}}$ for arbitrary values of $T_{23}$ and $T_{14}$, with the constraint $T_{14}-T_{23}=2 T$.

We note that the noise levels are significantly different, in particular at low frequency, illustrating the potential gain one can expect from using the optimal observable. In particular the observable $y_{\mathrm{r}}$ with $T_{23}=0$ used in "classical" Doppler ranging shows several orders of magnitude larger noise at low frequency than the timing observable $y_{\mathrm{t}}$ with $T_{23}=0$. This is due to the cancelation of the onboard accelerometer noise in (32) leaving only the onboard clock as the dominant noise contribution at low frequency. This advantage has to be weighed against the different sensitivity functions $b_{\mathrm{r}}$ and $b_{\mathrm{t}}$ as shown in fig. 3 in order to determine the optimal observable as a function of Fourier frequency, which will be the subject of the following section. 

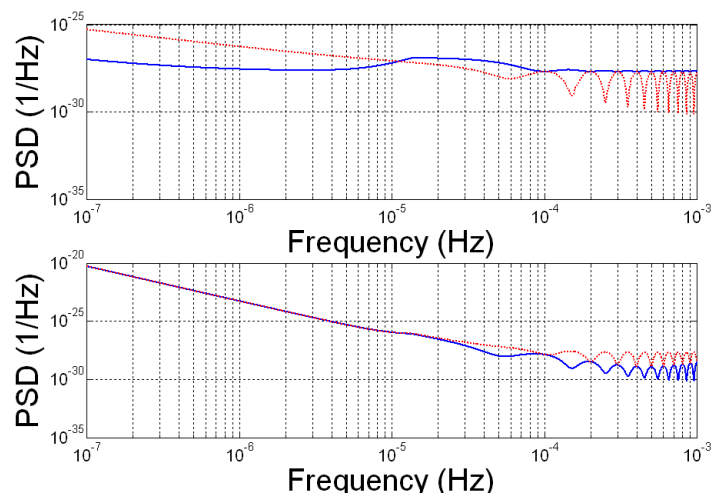

FIG. 5: PSD of the noise affecting the two observables $y_{\mathrm{t}}$ (upper graph) and $y_{\mathrm{r}}$ (lower graph) for the special cases $T_{23}=$ 0 (blue-solid) and $T_{14}=0$ (red-dotted), and for a spacecraft to ground distance of $10 \mathrm{AU}$.

\section{CONSTRAINTS ON GW BACKGROUNDS}

The case of ranging or Doppler tracking $\left(y_{\mathrm{r}}\right)$ has been discussed in numerous papers with the best bounds given by Doppler tracking of the Cassini probe [12, 30]. Oneway linking has also been studied since it is involved in the extremely impressive bounds derived from pulsar timing [38, 39]. Here we will focus our attention on the case of synchronization between remote clocks. As made clear by the discussion of the preceding section, this points to experiments with excellent clocks at large distances, and we will take the SAGAS project as an example. We begin by discussing a somewhat simplified case illustrating the advantages of the different observables and combinations, and then go on to derive limits using the complete SAGAS noise sources as discussed in section 4 .

In order to discuss the attainable performances, we use the spectra associated with time derivatives of the phaseshifts induced by GW backgrounds (eq. 9). These have to be compared to the phase variation induced by the noise sources discussed in section 4 . The equality of the two provides the obtainable upper limit of $S_{\mathrm{GW}}$

$$
S_{y}[\omega]=\omega^{2} S_{\tau}[\omega]=\frac{5}{8} b[\omega] S_{\mathrm{GW}}[\omega]
$$

with $S_{y}$ and $b$ having different forms for the different observables (cf. equations (23), (31), (32)).

For comparison with known bounds [18], we describe the gravitational noise in terms of the reduced gravitational energy density $\Omega_{\mathrm{GW}}$ commonly used to discuss the cosmic backgrounds $\left(H_{0} \simeq 71 \mathrm{kms}^{-1} \mathrm{Mpc}^{-1} \simeq\right.$ $2.3 \times 10^{-18} \mathrm{~Hz}$ is the Hubble constant)

$$
\Omega_{\mathrm{GW}}=\frac{10 \pi^{2} f^{3}}{3 H_{0}^{2}} S_{\mathrm{GW}}
$$

Collecting (33) and (34), we deduce the expression of the bound obtainable on $\Omega_{\mathrm{GW}}$ from that of the sensitivity function $b$ and the noise $S_{y}$

$$
\Omega_{\mathrm{GW}}=\frac{16 \pi^{2} f^{3}}{3 H_{0}^{2}} \frac{S_{y}}{b}
$$

\section{A. Illustration using a simplified case}

To illustrate how the obtainable limits can be optimised using the available measurements and resulting observables, we first consider an idealized case where only three noise sources play a significant role, the onboard clock and accelerometer, and the ground clock. Furthermore, we will assume that all three noises consist of only white noise, at the levels indicated by equations (26, 28 and 27), ie. we will only consider the first terms of those equations in the expressions (31, 32) for $S_{y}$, with all other terms set to zero.

Figure 6 shows the resulting limits on $\Omega_{\mathrm{GW}}$ as a function of frequency for two satellite to ground distances: $6 \mathrm{AU}$, the distance of Cassini when the GW experiment was carried out [30], and $53 \mathrm{AU}$, the maximum distance envisaged for the SAGAS mission [19]. Limits are shown for three observables: $\Omega_{\mathrm{r}}$ is obtained using the "classical" Doppler ranging observable as defined in (11) with $t_{2}=t_{3}$. We recall that this is the only observable available on space-probes equipped only with a transponder (the case of all probes flown so far). $\Omega_{\mathrm{t}}$ is obtained using the timing observable defined in (2) again with the condition $t_{2}=t_{3}$. $\Omega_{\text {opt }}$ is calculated by adjusting $T_{23}$ in (23. 31 and 32) for each frequency in order to minimize the obtained limit on $\Omega_{\mathrm{GW}}$.

In doing so, one can use either the ranging or timing combination, the obtained optimal limits being identical (albeit for different values of $T_{23}$ ). That property is the result of the periodic dependence of equations (23. 31 and 32) on $T_{23}$, which means that at any given frequency one can find two values of $T_{23}$ for which the ranging and timing combinations yield the same limit on $\Omega_{\mathrm{GW}}$ in equation (35). However, the assumption that the up and down travel times are similar (see section 3.1) limits the allowed range of $T_{23}$. Taking into account the maximum relative probe-earth velocity $(\approx 50 \mathrm{~km} / \mathrm{s})$ we limit $T_{23}$ in the calculation of $\Omega_{\mathrm{opt}}$ so that the up and down travel times do not differ by more than $1 \%$. We then choose as $\Omega_{\text {opt }}$ the lower of the two limits obtained from $y_{\mathrm{r}}$ and $y_{\mathrm{t}}$ with a free choice of $T_{23}$ within the $1 \%$ limit mentioned above.

The graphs on figure 6 can be understood qualitatively and quantitatively when considering the expressions for the sensitivity functions (23) and the overall noise of the observables (31) and (32) that enter into the calculation of $\Omega_{\mathrm{GW}}$ in (35). We first discuss $\Omega_{\mathrm{r}}$ and $\Omega_{\mathrm{t}}$ in the case $T_{23}=0$, and then come to $\Omega_{\mathrm{opt}}$.

At the low frequency limit $(\omega T<<1), b_{\mathrm{r}} \propto(\omega T)^{2}$ and $b_{\mathrm{t}} \propto(\omega T)^{4}$ (see section 3.2). The noise at low frequency is dominated by the space probe motion for $S_{y_{\mathrm{r}}}$ and is thus proportional to $\omega^{-2}$, but that contribution is 

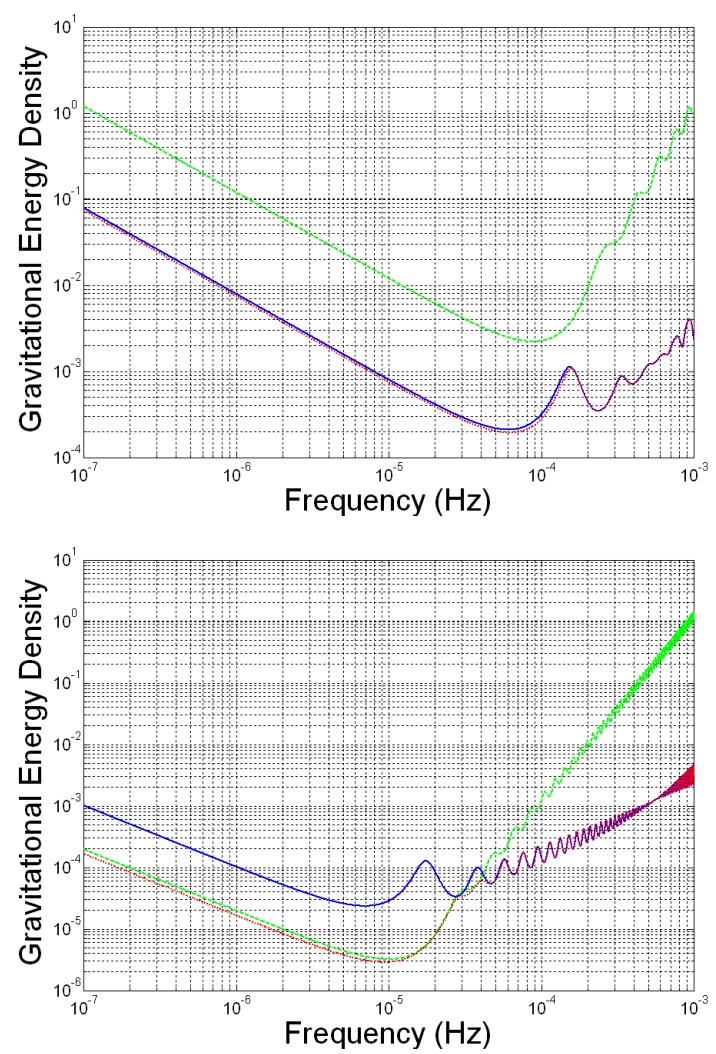

FIG. 6: $\Omega_{\mathrm{GW}}$ versus $f$, for a probe to Earth distance of $6 \mathrm{AU}$ (left) and $53 \mathrm{AU}$ (right). The three curves show the limits on $\Omega_{\mathrm{r}}$ (blue-solid), $\Omega_{\mathrm{t}}$ (green-dashed) and $\Omega_{\mathrm{opt}}$ (red-dotted).

entirely canceled in $S_{y_{\mathrm{t}}}$ because of the condition $T_{23}=0$ in (32), so $S_{y_{\mathrm{t}}} \propto \omega^{0}$ (clock noise only). This leaves an overall $\omega^{-1}$ dependence for both $\Omega_{\mathrm{t}}$ and $\Omega_{\mathrm{r}}$, clearly displayed in both plots of figure 6 at low frequency. Also, $\Omega_{\mathrm{r}}$ is significantly lower than $\Omega_{\mathrm{t}}$ at $6 \mathrm{AU}$, while the inverse is true at $53 \mathrm{AU}$ (at low frequency). This is caused by a tradeoff between the difference in sensitivity functions and the involved noise sources. We find more specifically and to leading order

$$
\lim _{\omega T \ll 1} \frac{\Omega_{\mathrm{r}}}{\Omega_{\mathrm{t}}}=\frac{1}{4}(\omega T)^{2} \frac{S_{v_{S}}}{S_{y_{S}}}
$$

where $S_{v_{S}}$ and $S_{y_{S}}$ are the first terms of (28) and (26) respectively, ie. $S_{v_{S}} / S_{y_{S}} \simeq 3 \times 10^{-8} \omega^{-2}$. Thus for large distances (greater than $\simeq 20$ AU with SAGAS figures) the low frequency asymptote is lower for timing than for ranging, leading to the observed inversion of $\Omega_{\mathrm{r}}$ and $\Omega_{\mathrm{t}}$ when passing from $6 \mathrm{AU}$ to $53 \mathrm{AU}$.

At the other end of the spectrum $(\omega T>>1)$, ranging outperforms timing (ie. $\Omega_{\mathrm{r}}<\Omega_{\mathrm{t}}$ ) at both distances. This can be easily understood when considering only the involved noise sources, as the sensitivity functions show oscillatory behavior and differ at most by a factor 3 (see section 3.2). At high frequency (between $10^{-4} \mathrm{~Hz}$ and $\left.10^{-3} \mathrm{~Hz}\right), S_{y_{\mathrm{t}}}$ is dominated by the space clock, but that contribution is entirely canceled in $S_{y_{\mathrm{r}}}$ because of the condition $T_{23}=0$ in (31), leaving only a combination of space probe motion and ground clock noise. As at high frequency the space clock noise is significantly higher than that of the space probe motion or the ground clock (see figure 4) this leads to the observed advantage of $\Omega_{\mathrm{r}}$ over $\Omega_{\mathrm{t}}$. Note also, the difference in slope between $\Omega_{\mathrm{r}}$ and $\Omega_{\mathrm{t}}$ at high frequency, particularly visible at $53 \mathrm{AU}$, which can be easily understood from the slopes of the spectra of the different noise contributions $\left(S_{y_{q}} \propto f^{0}\right.$, $\left.S_{v_{S} / c} \propto f^{-2}\right)$.

The lowest limits are obtained in the intermediate region $(\omega T \simeq 1)$ with very different results for the two distances. As expected, even when using only the "classical" ranging observable $\left(\Omega_{\mathrm{r}}\right.$ on figure 6), limits improve with distance by about the ratio of distances (about an order of magnitude in the present case). However, it is clearly seen that almost another order of magnitude can be gained when taking advantage of the timing observable $\left(\Omega_{\mathrm{t}}\right.$ in figure 6), only available when using space probes equipped with an onboard clock and a two-way electromagnetic link.

In that case, one can not only choose between $y_{\mathrm{r}}$ and $y_{\mathrm{t}}$, but also adjust the value of $T_{23}$ in order to optimize the measurement for any given frequency. The result of such an optimization, $\Omega_{\mathrm{opt}}$, is shown in figure 6. As expected it is below $\Omega_{\mathrm{r}}$ and $\Omega_{\mathrm{t}}$ at all frequencies and for both distances. Although the overall improvement is not spectacular, one obtains the "best of both worlds", in particular at $53 \mathrm{AU}$ where $\Omega_{\mathrm{opt}}$ follows $\Omega_{\mathrm{t}}$ at low frequency and $\Omega_{\mathrm{r}}$ at high frequency. We notice a slight improvement on $\Omega_{\mathrm{t}}$ at low frequency and $53 \mathrm{AU}$, which can be understood by considering the series expansion of the sensitivity function $b_{\mathrm{t}}$ in (23)). Additionally to the term in $(\omega T)^{4}$ present in the case $T_{23}=0$ (see section 3.2) one now obtains a term proportional to $(\omega T)^{2}\left(\omega T_{23}\right)^{2}$ which can be significantly larger. However, when $T_{23} \neq 0$, low frequency noise from the space probe motion is added, the trade off between the two leading to the small improvement of $\Omega_{\text {opt }}$ over $\Omega_{\mathrm{t}}$ seen on figure 6 .

In conclusion, the simplified case used in this section illustrates the advantages of having an onboard clock and a two-way link, which allows one to "fine tune" the data analysis as a function of the expected signal and the noise sources affecting the raw measurements. In this example, the sensitivity to GW backgrounds at large distance (53 AU) is improved by about an order of magnitude over the "classical" case $\Omega_{\mathrm{r}}$ by choosing the optimal combination of the available measurements on ground and onboard the space probe. Similar (up to a factor 20) improvements are observed when taking into account all noise sources discussed in section 4 .

\section{B. SAGAS limits on GW backgrounds}

We now repeat the calculations described in the previous section, using the example of SAGAS including all noise sources described in section 4. For clarity, we 
show only the resulting optimal limits $\Omega_{\mathrm{opt}}$ for a range of distances (see figure 77). The Big Bang Nucleosynthesis (BBN) bound, which corresponds to a flat floor with $\Omega_{\mathrm{BBN}} \sim 1.5 \times 10^{-5}$ has been drawn for comparison.

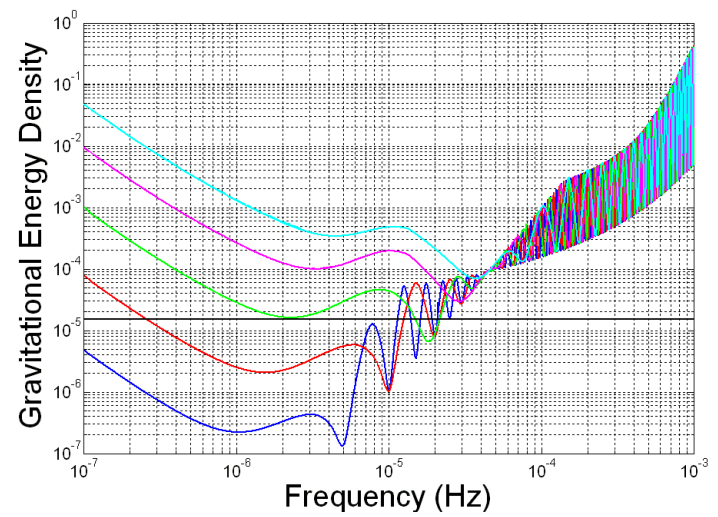

FIG. 7: $\Omega_{\mathrm{opt}}$ versus $f$, for probe to Earth distances (top to bottom) of $20 \mathrm{AU}$ (light blue), $30 \mathrm{AU}$ (magenta), $53 \mathrm{AU}$ (green), $100 \mathrm{AU}$ (red) and $200 \mathrm{AU}$ (dark blue). The horizontal black line indicates the BBN bound.

As expected, the sensitivity to GW is improved by going to large distances. The frequency at which the lowest limits are reached is typically in the region where $f T \sim 1$ ie. decreasing with increasing distance. For SAGAS (projected distance $\sim 53 \mathrm{AU}$ ) the lowest obtained limit is about $\Omega_{\mathrm{GW}} \leq 7 \times 10^{-6}$ around $2 \times 10^{-5} \mathrm{~Hz}$ and essentially determined from the timing observable. It is more than 3 orders of magnitude below the best directly measured limits in the $10^{-6}$ to $10^{-4} \mathrm{~Hz}$ band, obtained from the Cassini probe [30], and about a factor 2 below the BBN bound. At larger distances the timing measurement could even approach the pulsar bound around a few $10^{-8}$, but at significantly higher frequencies (the pulsar bounds are at a few $\mathrm{nHz}[38]$ ).

\section{Discussion}

Existing bounds on stochastic GW backgrounds are spread over a huge frequency range from $10^{-18} \mathrm{~Hz}$ to $10^{3} \mathrm{~Hz}$, corresponding to cosmological bounds obtained from measurements of the $3 \mathrm{~K}$ microwave background (COBE) at the lower end, and modern ground based GW detectors (LIGO, VIRGO) at the upper end (see e.g. figure 14 of [18]). This large frequency range is patchily covered, with COBE limits at $10^{-18}-10^{-16} \mathrm{~Hz}$, pulsar bounds around $10^{-9}-10^{-8} \mathrm{~Hz}$, spacecraft Doppler ranging covering three orders of magnitude $\left(10^{-6}-10^{-3}\right.$ $\mathrm{Hz}$ ), and GW detectors setting limits around $10^{2} \mathrm{~Hz}$. This is complemented by an indirect upper limit derived from models of BBN which corresponds to a flat floor of $\Omega_{\mathrm{GW}} \leq 1.5 \times 10^{-5}$ at all frequencies $\geq 10^{-10} \mathrm{~Hz}$. The latter is already outperformed by the pulsar limits at low frequency and is expected to be outperformed at high fre- quency by LIGO and VIRGO measurements in the near future.

In this landscape, limits obtained from spacecraft tracking play an important role as they fill a large part of the gap in frequency between the pulsar limits and those obtained from ground based detectors. Unfortunately, the obtained bounds are presently limited to $\Omega_{\mathrm{GW}} \leq 0.025[30]$. Improvements in this band will be particularly useful, especially when they will approach or surpass the BBN bound, as would be the case with future missions like SAGAS. As shown above, we expect that such missions could provide limits on $\Omega_{\mathrm{GW}}$ down to parts in $10^{-6}$ for SAGAS and below for missions at even larger distances.

More generally, it is important to obtain experimental constraints on $\Omega_{\mathrm{GW}}$ at all frequencies, as many of the models that predict such GW backgrounds are frequency dependent (eg. cosmic strings models, pre Big Bang models,...) and only poorly constrained in the presence of frequency gaps. In that respect the future space interferometric GW detector (LISA) plays an important role, as it should provide extremely low limits (down to $\Omega_{\mathrm{GW}} \leq 10^{-13}$ ) in the still largely unconstrained frequency range of $10^{-4} \mathrm{~Hz}$ to $10^{-1} \mathrm{~Hz}$. It should even be able to observe the astrophysical GW background from an ensemble of galactic binary stars, estimated to be too low for any other present or planned detector, but within the reach of LISA.

\section{CONCLUSION}

Doppler ranging to distant space probes provides the presently most stringent upper bounds on GW between $10^{-6}$ and $10^{-3} \mathrm{~Hz}$. Those bounds are obtained by "passive" ranging, where the space probe only serves as a "reflector" of the signal emitted from the ground. We have shown that the sensitivity can be significantly improved when having a clock onboard, so that the up and down signals are independent (asynchronous link) and can be combined in an optimal manner adapted to the signal to be measured and the noise affecting the link. We have derived explicit expressions for the sensitivity of all possible link combinations to a $\mathrm{GW}$ background. Using the example of the SAGAS project, we have evaluated the sensitivity of such a mission to GW backgrounds for optimal signal combinations and as a function of distance, with a potential improvement by over three orders of magnitude on best present limits.

Let us notice the similarities between the calculations of the present paper and those previously devoted to the effect of stochastic GW backgrounds on inertial sensors built on atomic interferometry [28, 40]. The sensors of interest in the present paper are the atomic clocks the indications of which are compared through electromagnetic links. As these links cannot be protected against the action of GW backgrounds, there exists an ultimate noise in clock synchronisation due to the presence of this 
universal fluctuating environment. It has been shown in the present paper that timing can be more sensitive to this environment than ranging, provided that extremely large distances are considered, as it is the case in the SAGAS project.

In our estimations we have chosen a conservative approach where the noise on the spacecraft motion is determined solely by the measurement noise of the onboard accelerometer. Previous deep space probes, in particular the Cassini mission [30], did not have an accelerometer on board, and all non-gravitational accelerations acting on the probe where determined by fitting acceleration models to the ranging data. The PSD of the residuals is most likely dominated by ground station and antenna motion at low frequency, and in particular around the diurnal frequency and its harmonics (see figure 1 of [30]). For a mission like SAGAS this suggests an analysis strategy based on the cancellation of the ground station motion rather than that of the space probe. In frequency regions where ground station noise is dominant one would use the timing observable (2) giving rise to $S_{y_{\mathrm{t}}}(f)$ of (32), but with the condition $T_{14} \simeq 0$ (coincidence of up and down signals at the ground antenna). As can be easily seen from (32), this leads to cancellation of noise from the ground station motion and the troposphere, leaving space clock instabilities and space probe motion as the dominant noise sources (see figure 4). To evaluate the limits obtained in this scenario requires a more detailed investigation of the space probe motion, the effect of fitting acceleration models, the improvements in the fits from in situ acceleration measurements, etc..., which are beyond the scope of this paper. Nonetheless, this alternative approach well illustrates the versatility of using an asynchronous link that allows choosing the optimal data combination strategy, even after launch, and as a function of the observed noise levels.

Finally, we point out that this data combination strategy can be adapted and optimized for any signal that is to be measured. The GW backgrounds discussed in this paper give rise to sensitivity functions (23) which enter the parameter $\Omega_{\mathrm{GW}}$ in (35) together with the noise (31), (32). That parameter is then optimized over a broad frequency range by varying $T_{23}$ or $T_{14}$. A similar procedure can be used for other science objectives (e.g. test of the gravitational time delay during occultation, measurements of planetary gravity, trajectory determination during fly by, etc...) by deriving the appropriate sensitivity functions and calculating the parameters to be optimized. It is likely to allow significant improvements in those measurements as well.

\section{Acknowledgments}

Helpful discussions with Jim Ray of NGS/NOAA concerning GNSS, SLR and VLBI positioning noise are gratefully acknowledged. Loic Duchayne acknowledges financial support from ESA, EADS-Astrium and CNES.
[1] Petit G. and Wolf P., Metrologia 42 S138 (2005)

[2] Shapiro I.I., Rev. Mod. Phys. 71 S41 (1999).

[3] Dickey J.O. et al, Science 265482 (1994)

[4] Williams J.G., Newhall X.X. and Dickey J.O., Phys. Rev. D53 6730 (1996)

[5] Blanchet L., Salomon C., Teyssandier P. and Wolf P., A.A. 370320 (2001)

[6] Anderson J.D., Gross M., Nordtvedt K. et al, Ap. J. 459 365 (1996)

[7] Estabrook F.B. and Wahlquist H.D., Gen. Rel. Grav. 6 439 (1975)

[8] Hellings R.W., in Detection of Gravitational Waves, ed. D. Blair (Cambridge University Press, Cambridge, 1992), p. 453

[9] Anderson J.D., Armstrong J.W., Estabrook F.B. et al , Nature 308158 (1984)

[10] Bertotti B., Vecchio A. and Iess L., Phys. Rev. D59 082001 (1999)

[11] Tinto M., Class. Quant. Grav. 191767 (2002)

[12] Armstrong J.W., Living Rev. Rel. 1 (2006), http://relativity.livingreviews.org/

[13] Giazotto A., Bonazzola S. and Gourgoulhon E. Phys. Rev. D55 2014 (1997)

[14] Grishchuk L.P. et al , Phys. Usp. 441 (2001)

[15] Hils D., Bender P.L. and Webbink R.F. Astrophys. J. 36075 (1990)

[16] Maggiore M., Phys. Rep. 331283 (2000)
[17] Schutz B., Class. Quant. Grav. 16 A131 (1999)

[18] Abbott B. and the LIGo collaboration, astro-ph/0608606

[19] Wolf P. and the SAgas team, arXiv:0711.0304

[20] Diddams S.A. et al , Science 3061318 (2004)

[21] Fortier T.M. et al, Phys. Rev. Lett. 98070801 (2007)

[22] Hoyt C.W. et al , Proc. 20th EFTF, p.324, Besançon, France, (2006)

[23] Petit G. and Wolf P., A.A. 286971 (1994); Wolf P. and Petit G., A.A. 304653 (1995)

[24] Le Poncin-Lafitte C., Linet B. and Teyssandier P., Class. Quantum Grav. 214463 (2004)

[25] Jaekel M.-T. and Reynaud S., Class. Quantum Grav. 23 777 (2006); Class. Quantum Grav. 237561 (2006)

[26] Asmar S.W., Armstrong J.W., Iess L. et al , Radio Science 40 RS2001 (2005)

[27] Jaekel M.-T. and Reynaud S., Phys. Letters A185 143 (1994); Quantum and Semiclassical Optics 7639 (1995)

[28] Reynaud S., Lamine B. and Jaekel M.-T., in Proceedings of the Varenna school on Atom Optics and Space Physics, to appear (Società Italiana di Fisica, 2008), arXiv:0801.3411 1 [gr-qc]

[29] Barnes J.A. et al , IEEE Trans. Instr. Meas. 20105 (1971)

[30] Armstrong J.W. et al , Ap. J. 599806 (2003)

[31] Van Camp M., Williams S.D.P. and Francis 0., J. Geophys. Res. 110 B05406 (2005)

[32] International GNSS Service http://igscb.jpl.nasa.gov/ 
[33] Larson K.M., Bilich A. and Axelrad P., J. Geophys. Res. 112 B05422 (2007)

[34] Ray J. et al, GPS Solutions, DOI 10.1007/s10291-0070067-7, (2007)

[35] Williams S.D.P. et al , J. Geophys. Res. 109 B03412 (2004)

[36] See for example the Colloquium on the Scientific and Fundamental Aspects of the Galileo Programme http://www. congrex.nl/07a06/

[37] Mendes V.B. et al, Geoph. Res. Lett. 291414 (2002); Mendes V.B. and Pavlis E.C., Geoph. Res. Lett. 31
L14602 (2004)

[38] Kaspi V.M., Taylor J.H. and Ryba M.F. Astrophys. J. 428713 (1994)

[39] Stairs I.H., Living Rev. Rel. 5 (2003), http://relativity.livingreviews.org/

[40] Lamine B., Jaekel M.-T. and Reynaud S., Eur. Phys. J. D20 165 (2002); Reynaud S., Lamine B., Lambrecht A. et al , Gen. Relat. Grav. 362271 (2004) Lamine B., Herv R., Lambrecht A. and Reynaud S., Phys. Rev. Lett. 96050405 (2006) 